\title{
$\beta$-Synuclein Aggregates and Induces Neurodegeneration in Dopaminergic Neurons
}

\author{
Grit Taschenberger, PhD, ${ }^{1}$ Johan Toloe, PhD, ${ }^{1}$ Julia Tereshchenko, PhD, ${ }^{1}$ \\ Jasper Akerboom, $\mathrm{PhD}_{1}^{2}$ Pauline Wales, $\mathrm{PhD},{ }^{3}$ Roland Benz, $\mathrm{PhD},{ }^{4,5}$ \\ Stefan Becker, PhD, ${ }^{6}$ Tiago F. Outeiro, PhD, ${ }^{3}$ Loren L. Looger, $\mathrm{PhD}{ }^{2}$ \\ Mathias Bähr, MD, ${ }^{1}$ Markus Zweckstetter, $\mathrm{PhD}^{6,7}$ and Sebastian Kügler, PhD ${ }^{1}$
}

Objective: Whereas the contribution of $\alpha$-synuclein to neurodegeneration in Parkinson disease is well accepted, the putative impact of its close homologue, $\beta$-synuclein, is enigmatic. $\beta$-Synuclein is widely expressed throughout the central nervous system, as is $\alpha$-synuclein, but the physiological functions of both proteins remain unknown. Recent findings have supported the view that $\beta$-synuclein can act as an ameliorating regulator of $\alpha$-synuclein-induced neurotoxicity, having neuroprotective rather than neurodegenerative capabilities, and being nonaggregating due to the absence of most of the aggregation-promoting NAC domain. However, a mutation of $\beta$-synuclein linked to dementia with Lewy bodies rendered the protein neurotoxic in transgenic mice, and fibrillation of $\beta$-synuclein has been demonstrated in vitro.

Methods: Neurotoxicity and aggregation properties of $\alpha_{-}, \beta-$, and $\gamma$-synuclein were comparatively elucidated in the rat nigro-striatal projection and in cultured neurons.

Results: Supporting the hypothesis that $\beta$-synuclein can act as a neurodegeneration-inducing factor, we demonstrated that wild-type $\beta$-synuclein is neurotoxic for cultured primary neurons. Furthermore, $\beta$-synuclein formed proteinase K-resistant aggregates in dopaminergic neurons in vivo, leading to pronounced and progressive neurodegeneration in rats. Expression of $\beta$-synuclein caused mitochondrial fragmentation, but this fragmentation did not render mitochondria nonfunctional in terms of ion handling and respiration even at late stages of neurodegeneration. A comparison of the neurodegenerative effects induced by $\alpha-, \beta-$, and $\gamma$-synuclein revealed that $\beta$-synuclein was eventually as neurotoxic as $\alpha$-synuclein for nigral dopaminergic neurons, whereas $\gamma$-synuclein proved to be nontoxic and had very low aggregation propensity.

Interpretation: Our results suggest that the role of $\beta$-synuclein as a putative modulator of neuropathology in aggregopathies like Parkinson disease and dementia with Lewy bodies needs to be revisited.

ANN NEUROL 2013;74:109-118

T he synucleins are a family of small natively unfolded proteins, characterized by a highly conserved $\mathrm{N}$-terminus with 6 imperfect repeats and a less well-conserved acidic C-terminus. ${ }^{1} \alpha$-Synuclein $(\alpha S)$ and $\beta$-synuclein $(\beta S)$ are widely expressed throughout the central nervous system, whereas $\gamma$-synuclein $(\gamma S)$ is more prominently expressed in the peripheral nervous system. ${ }^{2,3}$ Despite their high abundance, the function of all 3 synucleins is largely unknown. Even triple knockout mice display only a mild phenotype of impaired neurotransmitter release and vesicle recycling in the dopaminergic system. ${ }^{4} \alpha S$ is intimately linked to Parkinson disease (PD) and related

View this article online at wileyonlinelibrary.com. DOI: 10.1002/ana.23905

Received Oct 9, 2012, and in revised form Mar 7, 2013. Accepted for publication Mar 22, 2013.

Address correspondence to Dr Kügler, University Medical Center Göttingen, Department of Neurology, Waldweg 33, 37073 Göttingen, Germany.

E-mail: sebastian.kuegler@med.uni-goettingen.de

From the ${ }^{1}$ Department of Neurology, Center for Nanoscale Microscopy and Molecular Physiology of the Brain at University Medical Center Göttingen, Göttingen, Germany; ${ }^{2}$ Janelia Farm Research Campus, Howard Hughes Medical Institute, Ashburn, VA; ${ }^{3}$ Department of Neurodegeneration and Restorative Research, University Medical Center Göttingen, Göttingen, Germany; ${ }^{4}$ Rudolf Virchow Center, DFG Research Center for Experimental Biomedicine, University of Würzburg, Würzburg, Germany; ${ }^{5}$ School of Engineering and Science, Jacobs University Bremen, Bremen, Germany; ${ }^{6}$ Max Plank Institute for Biophysical Chemistry, Göttingen, Germany; and ${ }^{7}$ Center for Neurodegenerative Diseases, Göttingen, Germany. 
synucleinopathies as a major constituent of Lewy bodies (LBs) and Lewy neurites (LNs) and high penetrance in the few familial cases caused by the $A 30 P, A 53 T$, and $E 46 K$ mutations or gene dosage duplications or triplications. ${ }^{5}$ In contrast, no genetic connection of $\beta S$ and $\mathrm{PD}$ has been described so far, and $\beta S$ is not detectable in LBs and LNs. Wild-type $\beta$ S has been shown to act as a scavenger for $\alpha S$ aggregation and neurotoxicity in a variety of experimental systems in vitro and in vivo. ${ }^{6-9}$ Under some conditions where $\alpha S$ and $\gamma S$ form fibrils, $\beta S$ did not fibrillate, and both $\beta S$ and $\gamma S$ inhibited fibril formation of $\alpha \mathrm{S} .{ }^{6}$ However, macromolecular crowding, metal ions, and pesticides readily lead to fibrillation of $\beta S,{ }^{10}$ demonstrating its conversion into a potentially neurodegeneration-inducing protein by environmental toxins, a condition closely associated with PD. ${ }^{11}$ Although $\beta S$ and $\gamma S$ are not yet directly linked to neurodegenerative processes in the ageing brain, their contribution to major neurological diseases has to be seriously considered. Both proteins can impact on structural properties of $\alpha S$, and have long been known as constituents of axonal lesions in hippocampus associated with $\mathrm{PD}$ and dementia with LBs (DLB). ${ }^{12}$ The DLB-linked $\mathrm{P} 123 \mathrm{H}$ mutation of $\beta S$ induced progressive neurodegenerative effects in transgenic $(\mathrm{tg})$ mice, which were strongly exacerbated by crossing with $\alpha \mathrm{S}$ tg mice. These double-tg mice demonstrated significantly enhanced loss of dopaminergic markers as compared to each single-tg line, suggesting that $\mathrm{P} 123 \mathrm{H}-\beta \mathrm{S}$ can directly cause neuronal lesions and that alterations in $\beta S$ levels can have influence in a wider range of synucleinopathies. ${ }^{13}$ Here we show that $\beta S$ expression directly leads to the formation of proteinase $\mathrm{K}(\mathrm{PK})$-resistant aggregates and progressive neurodegeneration in nigral dopaminergic neurons, eventually to the same extent as $\alpha$ S. In cultured neurons, $\beta S$ resulted in structural deterioration of mitochondria, but in terms of ion handling and respiration these organelles remained functional even at late stages of degeneration. This suggests that mitochondrial fragmentation may not be a causative insult for $\beta S$-induced neurodegeneration, despite our finding that $\beta S$ forms ionconductive pores in membranes. Our results demonstrate a direct neurotoxicity of $\beta S$ for nigral dopaminergic neurons, implying that not only $\alpha S$ but also $\beta S$ has to be considered as a toxic entity in PD, DLB, and other synucleinopathies.

\section{Materials and Methods}

\section{Recombinant Viruses}

Recombinant adeno-associated virus (AAV) vectors were prepared essentially as described ${ }^{14}$ and as detailed in the Supplementary Information. The genome layout of monocistronic vectors expressing human $\alpha \mathrm{S}, \beta S$, or $\gamma S$ or the reporter enhanced green fluorescent protein (EGFP) and of bicistronic vectors coexpressing the synucleins with EGFP is shown in Supplementary Figure 1A.

\section{Animal Procedures}

All experimental animal procedures were conducted according to approved experimental animal licenses, issued by the responsible animal welfare authority (Niedersächsisches Landesamt für Verbraucherschutz und Lebensmittelsicherheit) and controlled by the local animal welfare committee and veterinarians at the University Medical Center Göttingen essentially as described ${ }^{15}$ and as detailed in the Supplementary Information.

\section{Tissue Processing (Stereology, Immunohistochemistry, PK Digest)}

Detection of fibrillated synuclein by digestion of tissue specimen with PK, immunohistochemical detections, and stereological quantification of surviving nigral dopaminergic neurons were performed exactly as described elsewhere in detail. ${ }^{16}$ Antibodies used are described in the Supplementary Information.

\section{Neuronal Cell Culture, Respiration Measurement, and $\mathrm{Ca}^{2+}$ Imaging}

Primary cortical neurons were prepared from embryonic day 18 rat pups as described, ${ }^{17}$ and $\mathrm{Ca}^{2+}$ imaging was performed essentially as described, ${ }^{18}$ using red fluorescent mitochondriatargeted RCaMP1e as a sensor. Respiration rates were measured on a FX24 Extracellular Flux Analyzer (Seahorse Bioscience, North Billerica, MA) according to the manufacturer's instructions (see the Supplementary Information for details).

\section{Aggregation of $\beta \mathbf{S}$ and Membrane Assays}

$\beta S$ for aggregation and membrane assays was produced as described. ${ }^{19}$ A detailed description of conditions for $\beta$ S aggregation, oligomer isolation, and channel activity in artificial diphytanoyl-phosphatidylcholine membranes is given in the Supplementary Materials and Methods.

\section{Statistical Analysis}

Multiple comparisons were made by 1-way analysis of variance followed by Tukey honestly significant difference test. The unpaired $t$ test with Welch correction was used for statistical comparison between 2 groups. Differences were considered significant at $p<0.05$. Statistical tests were performed using the $\mathrm{R}$ software package. ${ }^{20}$

\section{Results}

Neurotoxicity of Synucleins in Primary Neurons We first assessed the neurotoxicity of the synucleins in cultures of primary cortical neurons. Neurons were transduced with AAV- 6 vectors expressing either EGFP, $\alpha S$ plus EGFP, $\beta S$ plus EGFP, or $\gamma S$ plus EGFP, under control of the neuron-specific synapsin 1 gene promoter (see Supplementary Fig 1). Expression levels of $\alpha S$ and $\beta S$ were found to be equal in cultured neurons by Western 


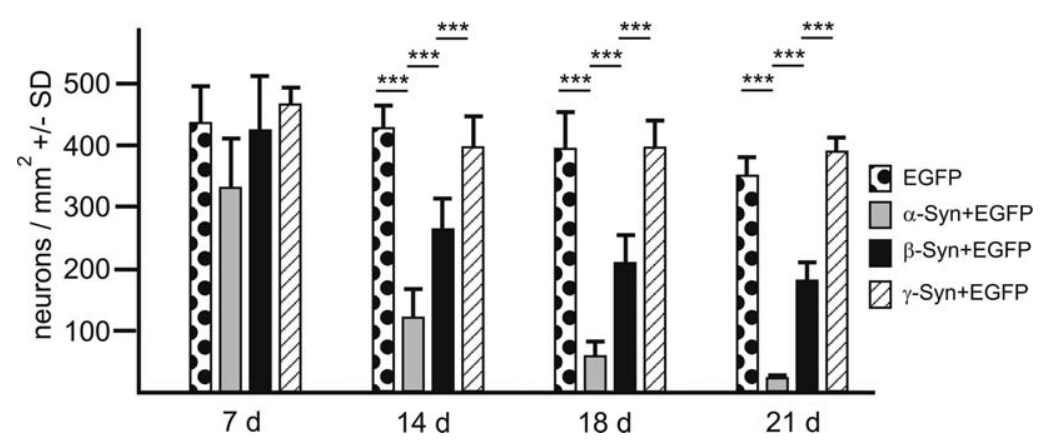

FIGURE 1: Neurotoxicity of synucleins (Syn) in cultured primary neurons. Primary cortical neurons were transduced at 3 days in vitro with the respective bicistronic adeno-associated virus vectors coexpressing enhanced green fluorescent protein (EGFP) with the synucleins $\left(1 \times 10^{9}\right.$ vector genomes/250,000 neurons/well). EGFP fluorescent neurons in living cultures and NeuNstained cells in fixed cultures were quantified at the indicated times after transduction (see Supplementary Fig 2 for details). Dotted bars = neurons expressing EGFP only, light gray bars = neurons expressing $\alpha$-synuclein and EGFP, black bars $=$ neurons expressing $\beta$-synuclein and EGFP, hatched bars $=$ neurons expressing $\gamma$-synuclein and EGFP. Statistics were performed by 1 -way analysis of variance/Tukey $\left({ }^{* *} p<0.001 ; n=6\right.$ independent transductions per condition). SD $=$ standard deviation.

blotting with an anti-pan-synuclein antibody detecting an identical epitope in $\alpha \mathrm{S}$ and $\beta S$. $\gamma S$ was detected with much less intensity, probably due to there being 2 different amino acids in the antigenic epitope. Thus, we also expressed V5 epitope-tagged synucleins and found equal expression levels by using the anti-V5 antibody, indicating that the reduced staining intensity of $\gamma S$ did not result from, for example, increased degradation of this synuclein isoform. In addition, isoform-specific antibodies showed very similar staining intensities on both denaturing and native blots.

As detailed in Figure 1 and Supplementary Figure 2, no significant neuron loss was observed up to 7 days after transduction, although transgene expression was detectable by EGFP fluorescence as early as 2 days after transduction. Between 10 and 14 days after transduction, prominent cell death occurred in the $\alpha S$ and, less prominently, in the $\beta S$-expressing neurons. Neurodegeneration continued through the next week in the $\alpha S$ neurons, leading to almost complete neuron loss. In $\beta S$-expressing neurons, cell loss did not proceed further. However, these $\beta S$-expressing neurons were clearly metabolically impaired, as their EGFP fluorescence did not increase further over time, as was observed in neurons expressing EGFP only or $\gamma S$ plus EGFP. Almost no neuron loss was observed in these latter cultures over the 3 weeks of the experiment. Coexpression of low level $\beta S$ partially ameliorated $\alpha S$-induced toxicity, but only if $A A V-\beta S$ was used in a $1: 30$ or $1: 10$ ratio to $A A V-\alpha S$, indicating that the ratio of both proteins can influence the degenerative phenotype (Supplementary Fig 3). These results revealed an unambiguous neurotoxicity of wild-type $\beta S$ in nondopaminergic primary neurons, whereas $\gamma S$ overexpression did not result in detectable neurodegeneration.

\section{Neurotoxicity of Synucleins in Dopaminergic Neurons In Vivo}

We next aimed to investigate the differential neurotoxicity of the synucleins in a paradigm more relevant for $\mathrm{PD}$. To this end, monocistronic AAV-2 vectors expressing EGFP, $\alpha S, \beta S$, or $\gamma S$ were injected unilaterally into the substantia nigra of adult rats to study the degeneration in dopaminergic neurons, which constitutes a major hallmark of PD. Stereological quantification of vesicular monoamine transporter type 2-positive cells already revealed a significant loss of dopaminergic neurons through $\beta S$ expression at 2 weeks after vector injection (20.4\% lost dopaminergic neurons; Fig 2). This cell loss was less prominent as compared to $\alpha \mathrm{S}$-induced degeneration $(46 \%)$, thereby reflecting the results obtained in cultured neurons. At 8 weeks after vector injection, $\beta S$ induced neurotoxicity caused almost $45 \%$ of dopaminergic neurons to degenerate, which was the same extent of degeneration as induced by $\alpha \mathrm{S}$ overexpression. No cell loss occurred in the EGFP-expressing nigrae, and despite the long-term overexpression, no dopaminergic neuron loss was observed in $\gamma S$-expressing nigrae. Immunohistochemical staining of tissue sections with the pan-synuclein antibody revealed equal levels of fluorescent signals for all 3 synucleins, confirming their very similar expression levels (see Supplementary Fig 1F-N). Evidently, the pan-synuclein antibody can detect the nonlinearized epitope of the 3 isoforms with equal sensitivity.

The level of overexpression was estimated by immunohistochemistry to be 7- to 10 -fold as compared to the endogenous level of synucleins (see Supplementary Fig $1 \mathrm{O}-\mathrm{S})$, corresponding well to the roughly 5-fold overexpression level as determined by Western blotting (Supplementary Fig 4). These results demonstrated that wild-type $\beta S$ can induce substantial neurodegeneration in 

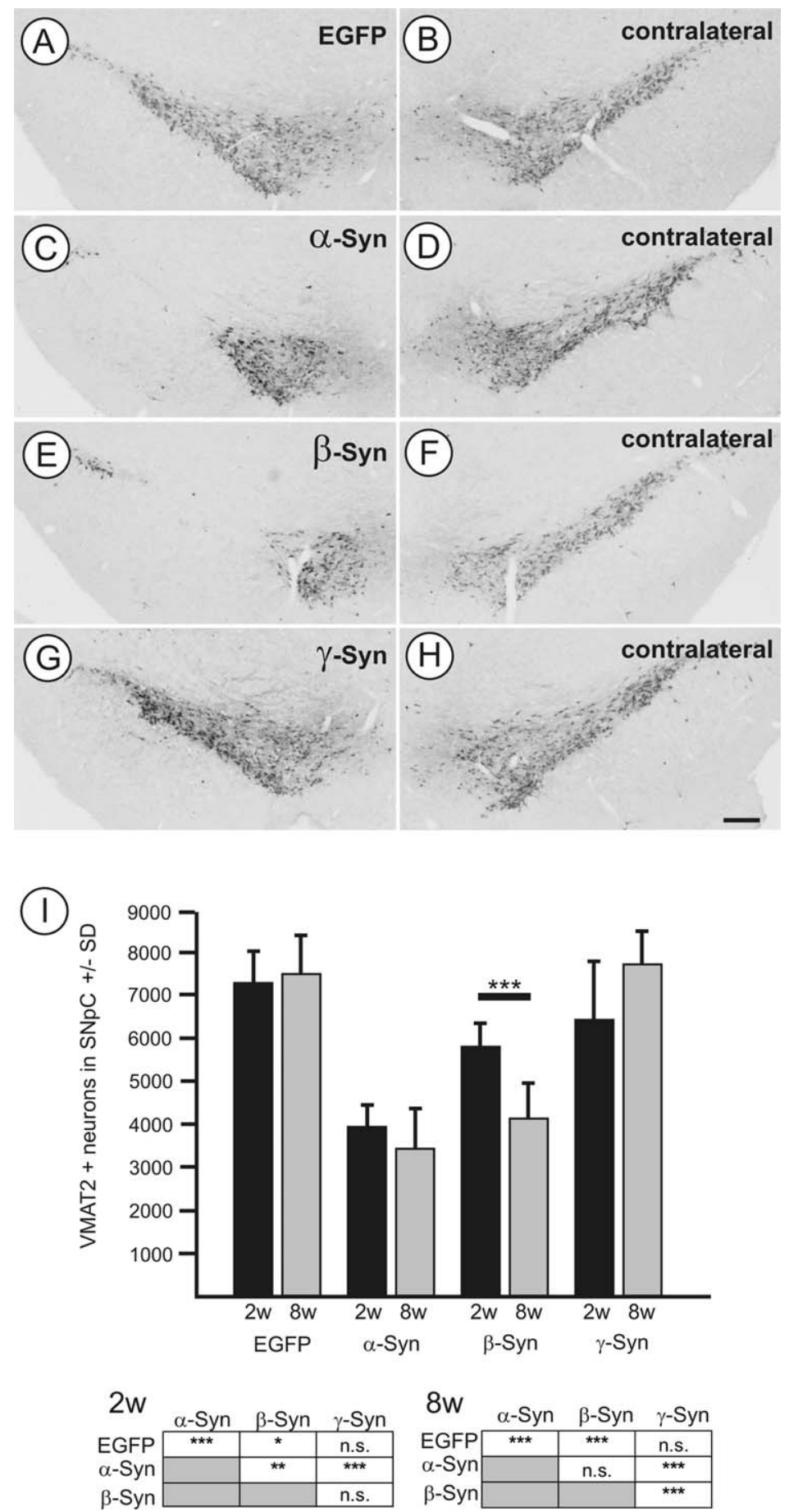

\begin{tabular}{|c|c|c|c|}
\hline $8 w$ & $\alpha-\operatorname{Syn}$ & $\beta$-Syn & $\gamma$-Syn \\
\hline \multirow{3}{*}{$\begin{array}{l}\text { EGFP } \\
\alpha \text {-Syn } \\
\beta-S y n\end{array}$} & $\star \star \star$ & 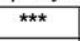 & n.s. \\
\hline & & n.s. & 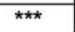 \\
\hline & & & $* \star \star \star *$ \\
\hline
\end{tabular}

FIGURE 2: (A-H) Neurotoxicity of synucleins (Syn) in nigral dopaminergic neurons in vivo. Vesicular monoamine transporter type 2 (VMAT2)-immunoreactive nigral neurons are shown at 8 weeks (w) after unilateral transduction with adeno-associated virus (AVV)-enhanced green fluorescent protein (EGFP; A, B), AAV- $\alpha$-synuclein (C, D), AAV- $\beta$-synuclein $(E, F)$, and AAV- $\gamma$ synuclein (G, H). (I) Stereological quantification of VMAT2-immunoreactive cells at 2 weeks after transduction (black bars) and at 8 weeks after transduction (gray bars). Statistics by 1-way analysis of variance/Tukey are given in the table below the diagram ( ${ }^{\star} p<0.05,{ }^{* \star} p<0.01,{ }^{\star \star *} p<0.001 ; n=5-7$ animals per condition). Scale bar $=200 \mu \mathrm{m}$. n.s. $=$ not significant; SD $=$ standard deviation; $\mathrm{SNpC}=$ substantia nigra pars compacta.

dopaminergic neurons in vivo. This neuron loss showed a significant progression between 2 and 8 weeks. Compared to $\alpha$, the neurotoxicity of $\beta S$ was less severe at the early time point but did not differ significantly at the late time point. Somewhat surprisingly, striatal pathology differed markedly between $\beta S$-expressing and $\alpha \mathrm{S}$-expressing brains, in that $\alpha S$ caused a more pronounced loss of dopaminergic axons (Supplementary Fig 5). 

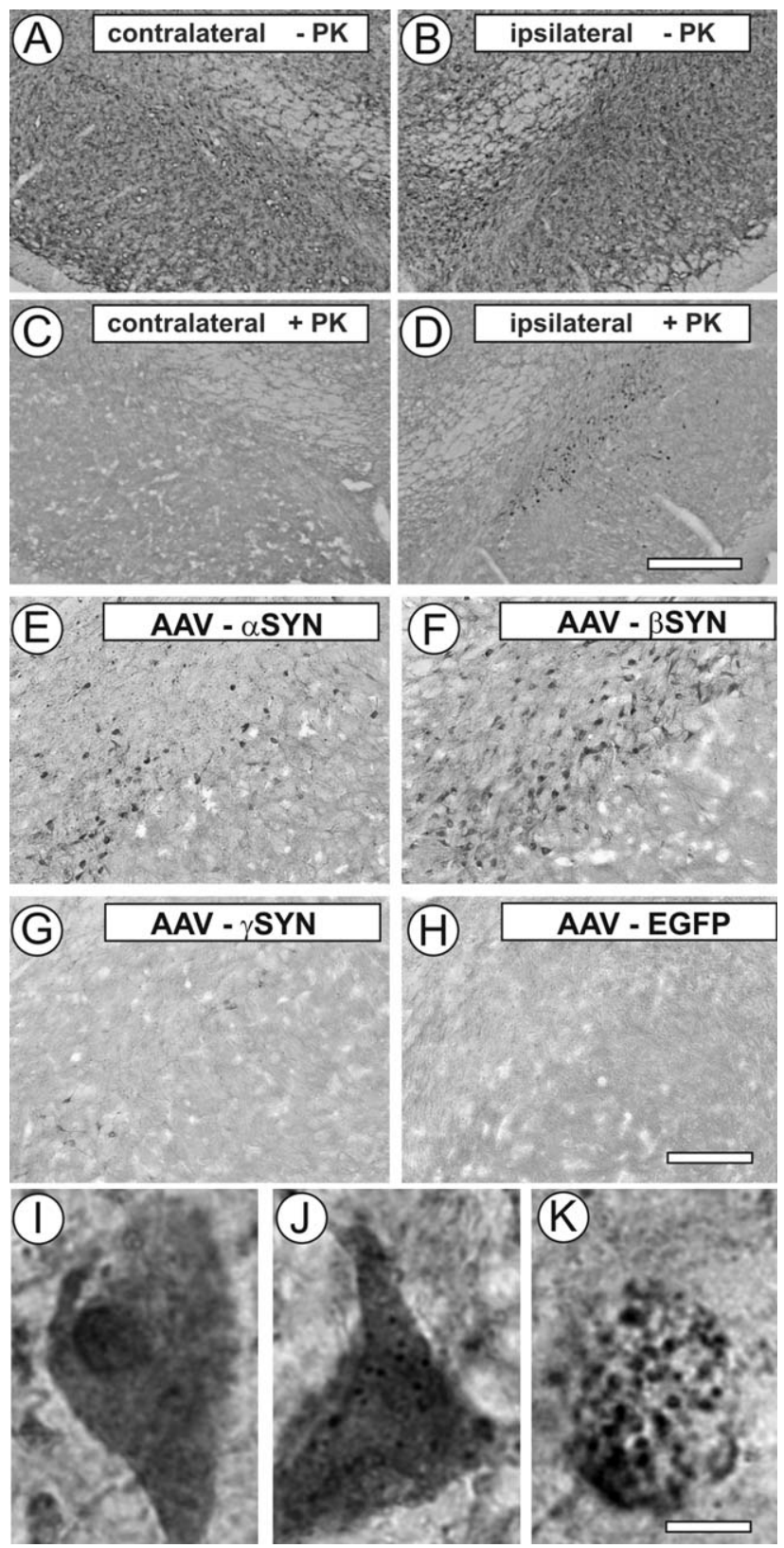

FIGURE 3: $\beta$-synuclein forms proteinase $\mathrm{K}$ (PK)-resistant aggregates in dopaminergic neurons. (A-D) Immunoreactivity to the anti-pan-synuclein (SYN) antibody is shown in untreated tissue sections for the contralateral side (A) and the adeno-associated virus (AAV)- $\beta$-synuclein-injected midbrain (B) and in PK-treated adjacent tissue sections for the contralateral side (C) and AAV$\beta$-synuclein-injected midbrain (D) at low magnification, demonstrating PK-resistant synuclein only in transduced neurons. (E-H) Prominent amounts of PK-resistant synuclein were detected after $\alpha$-synuclein expression (E) and $\beta$-synuclein expression (F), whereas minor amounts of PK-resistant synuclein were detected after $\gamma$-synuclein expression (G), and no PK-resistant synuclein was detectable after enhanced green fluorescent protein (EGFP) expression (H). (I-K) High-magnification micrographs of $\beta$-synuclein-expressing dopaminergic neurons after PK digestion showed either mostly homogenous staining in cytoplasm (I), additional small punctate aggregates in cells morphologically intact $(\mathrm{J})$, or massive accumulations of punctate aggregations in cells with disintegrated morphology (K). Scale bars $=500 \mu \mathrm{m}$ in $\mathrm{A}-\mathrm{D}, 125 \mu \mathrm{m}$ in $\mathrm{E}-\mathrm{H}$, and $10 \mu \mathrm{m}$ in $\mathrm{I}-\mathrm{K}$.

\section{Aggregate Formation of Synucleins in Dopaminergic Neurons In Vivo}

In vitro aggregation of $\beta S$ resulted in amyloid fibrils with high $\beta$-sheet content (Supplementary Fig 6A, B). Likewise, already at 2 weeks after vector injection we detected considerable amounts of PK-resistant synuclein in dopaminergic neurons after $\beta S$ expression (Fig 3). Staining intensity for these aggregates was similar to that in surviving $\alpha S$-expressing neurons, indicating early and substantial aggregation/fibrillation of $\beta S$ in vivo. In contrast, $\gamma S$-expressing neurons showed only very minor PKresistant deposits at 2 weeks after vector injection. These 
stainings were performed with the anti-pan-synuclein antibody that detects all three synucleins in tissue specimen, leaving open the possibility that $\beta S$ expression provoked an aggregation of endogenous $\alpha \mathrm{S}$. This was excluded by staining adjacent tissue sections with antibodies specific for $\alpha \mathrm{S}$ or $\beta S$, revealing that PK-resistant material detected in $\beta S$-expressing dopaminergic neurons was indeed $\beta S$, as no $\alpha \mathrm{S}$-immunoreactive dopaminergic neurons could be detected in AAV- $\beta$ S transduced brains (see Supplementary Fig 7). We detected no immunopositive PK-resistant material in AAV-EGFP-transduced nigrae, proving specificity of the respective antibodies.

In morphologically intact neurons, PK-resistant $\beta S$ was detectable either as a homogenously distributed cytoplasmic and perinuclear immunoreactivity (see Fig 3I) or with additional punctate accumulations (see Fig 3J). In cells with apparent disintegrated morphology, granular aggregates represent the major detectable immunoreactivity (see Fig 3K). It seems likely that these different aggregation states represent a temporal continuum.

In brains evaluated at 8 weeks after $\mathrm{AAV}$ vector injection, we detected strong labeling of PK-resistant $\alpha S$ and $\beta S$ in both neurites and cell bodies of surviving nigral neurons (Supplementary Fig 8A, B). In contrast, staining for PK-resistant synuclein was restricted to few cells with very faint staining intensity in $\gamma S$-expressing nigrae (see Supplementary Fig 8C), indicating that this protein indeed does not form fibrillated deposits to a significant extent. Of note, the very low level of immunoreactivity after $\mathrm{PK}$ digestion in $\gamma S$-expressing nigrae was detected with both the anti-pan-synuclein antibody and the $\mathrm{C}$-terminal-specific anti- $\gamma \mathrm{S}$ antibody, indicating that this lower level of detection was not due to impaired detection properties of the exploited antibody. These findings strongly correlated the aggregation propensity of the individual synucleins to their neurotoxicity, in that expression of $\alpha \mathrm{S}$ and $\beta \mathrm{S}$, which form PKresistant aggregates, resulted in pronounced degeneration of nigral dopaminergic neurons, whereas expression of $\gamma S$, which did not form PK-resistant aggregates, did not lead to detectable degeneration of nigral dopaminergic neurons.

\section{Mitochondrial Pathology Induced by $\beta$ S}

Mitochondria are deeply involved in the etiology and progression of PD and other aggregopathies. ${ }^{21,22}$ Importantly, interactions of $\alpha \mathrm{S}$ with mitochondria have been documented. $^{23}$ Thus, we aimed to assess whether $\beta S$ would have specific effects on mitochondrial functionality in living neurons in situ, which could explain the neurotoxicity of the protein. Structural mitochondrial deterioration was clearly detectable in AAV-transduced primary neurons expressing $\alpha \mathrm{S}$ and $\beta \mathrm{S}$ by live imaging (Fig 4). Mitochondria within neurons were classified qualitatively as either fully intact and tubular, partially condensed with many small-sized mitochondria, or fully condensed with a majority of swollen and rounded mitochondria. At 13 days of overexpression, the majority of surviving neurons showed destroyed mitochondria after $\alpha \mathrm{S}$ expression. At the same time, $50 \%$ of $\beta S$-expressing neurons contained destroyed mitochondria.

The capacity of mitochondria to actively remove elevated $\mathrm{Ca}^{2+}$ levels from their matrix is an indicator of sufficient energy supply and membrane potential integrity. ${ }^{24}$ As shown in Figure $4 \mathrm{E}$ and $\mathrm{F}$, both the peak $\mathrm{Ca}^{2+}$ influx and the decay to release $\mathrm{Ca}^{2+}$ from mitochondria were not significantly different between EGFPexpressing neurons and neurons expressing any of the synucleins. Furthermore, no significant difference between tubular, partially fragmented, and fully fragmented mitochondria was observed, indicating that even at states of advanced condensation mitochondria can still handle ion homeostasis appropriately.

Mitochondrial oxygen consumption was assessed by bioenergetic profiling. At 6 days after transduction no differences in oxygen consumption rate (OCR) could be detected between EGFP and synuclein-expressing neurons. At 13 days after transduction, measurement of basal respiration clearly demonstrated that in $\alpha S$ - and $\beta S$ expressing neurons, OCR was significantly lower as compared to EGFP- or $\gamma S$-expressing neurons (see Fig 4G). This decrease in basal respiration reflects the ongoing neurodegeneration at this time point. However, neither coupling efficacy nor the membrane potential-driven proton leak was obviously impaired by $\alpha \mathrm{S}$ or $\beta \mathrm{S}$, but due to high basic respiration with only moderate spare respiratory capacity, the fluoro-carbonyl cyanide phenylhydrazone-determined coupling efficacy has to be interpreted with caution. When these differences are addressed as changes relative to basal respiration (see Fig $4 \mathrm{H}$ ), they were only of a very moderate nature, suggesting that the capacity of mitochondria to produce energy is not robustly impaired by expression of any synuclein. However, at 13 days of overexpression, motile mitochondria in neurites of $\alpha \mathrm{S}$-expressing neurons, but not of $\beta S$ expressing neurons, demonstrated a significantly decreased velocity (see Fig 4I). This indicates that the primary impact of $\alpha \mathrm{S}$ may be more on the neuritic transport capabilities of mitochondria than on their direct functional destruction, which is supported by our finding of a more pronounced axonal degeneration in striata of $\alpha$ S-overexpressing rats (see Supplementary Fig 5). Overall, these results suggested that despite the mitochondrial fragmentation induced by $\beta S$ overexpression, 

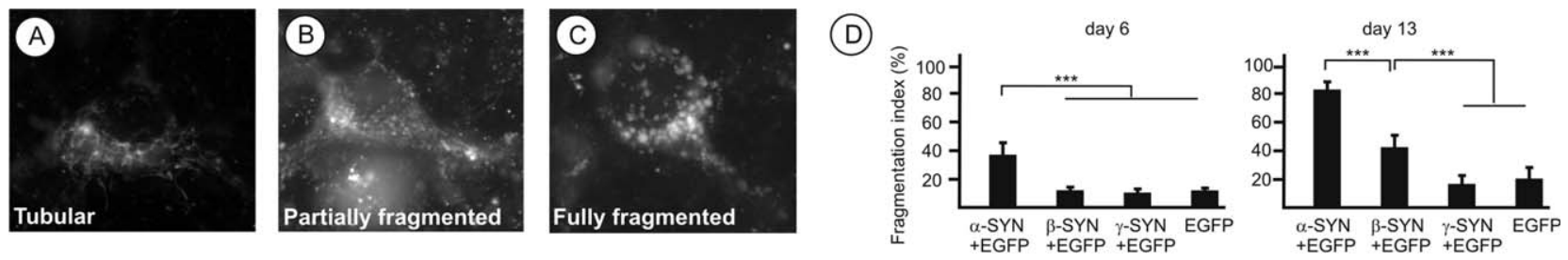

(E)

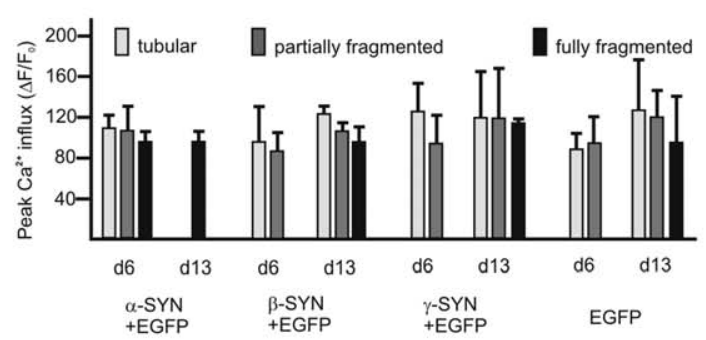

(F)
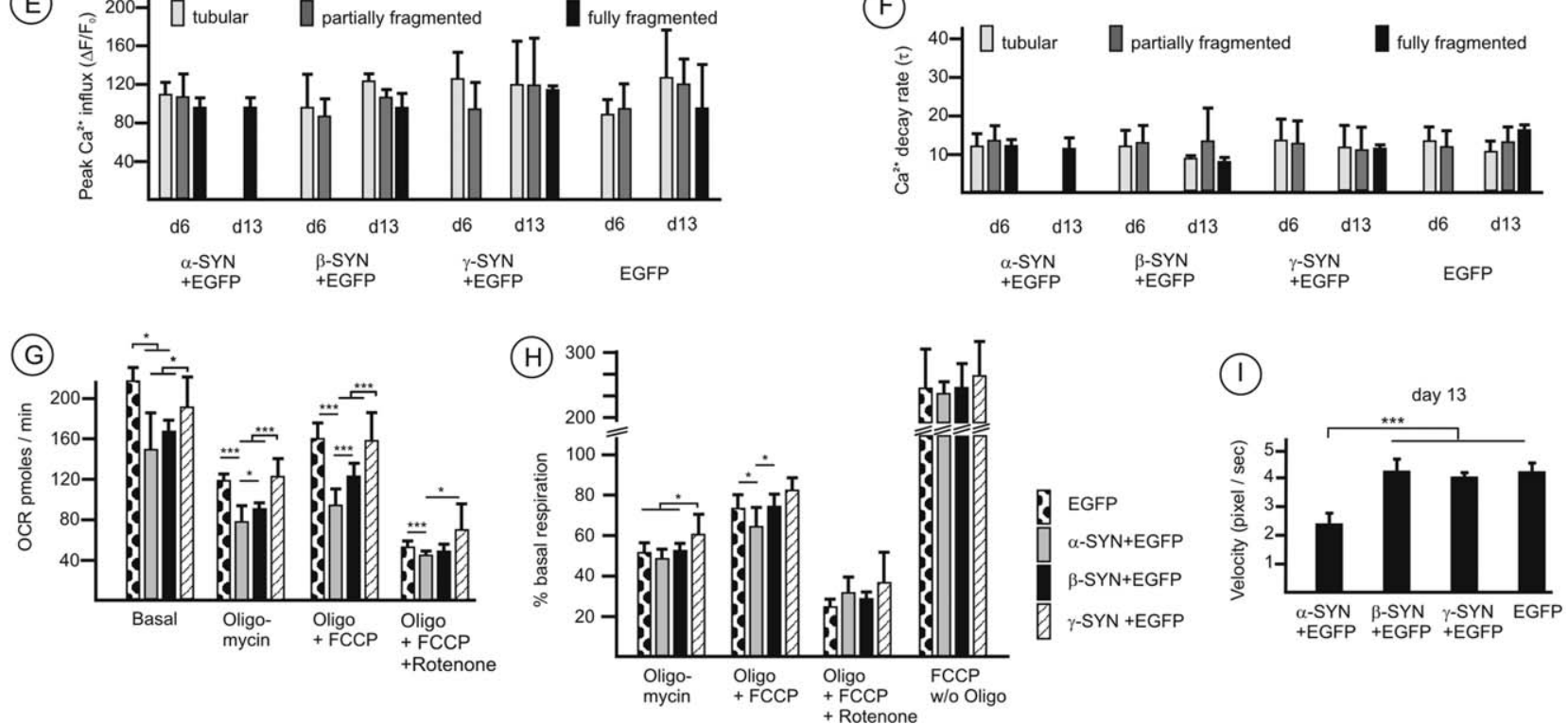

FIGURE 4: Mitochondrial pathology induced by the synucleins (SYN). Cultures of cortical neurons transduced with adeno-associated virus (AAV) vectors expressing enhanced green fluorescent protein (EGFP), $\alpha$-synuclein + EGFP, $\beta$-synuclein + EGFP, or $\gamma$ synuclein + EGFP were analyzed for mitochondrial fragmentation (A-D), mitochondrial $\mathrm{Ca}^{2+}$ handling $(E, F)$, mitochondrial oxygen consumption (G, H), and mitochondrial motility (I). Mitochondrial fragmentation index was calculated as the percentage of neurons containing a majority of fully fragmented mitochondria (C) at days 6 and 13 after transduction of neurons with the respective AAV vectors (D). Field stimulation of cultured neurons coexpressing either $\alpha$-synuclein, $\beta$-synuclein, $\gamma$-synuclein, or EGFP with a mitochondria-targeted red fluorescent genetically encoded $\mathrm{Ca}^{2+}$ sensor, mt-RCaMP1e, was used to study $\mathrm{Ca}^{2+}$ handling by quantifying the influx of $\mathrm{Ca}^{2+}$ into mitochondria after action potentials and the time necessary to pump this $\mathrm{Ca}^{2+}$ out of the mitochondria. Peak $\mathrm{Ca}^{2+}$ influx (E) and decay time (F) after field stimulation were quantified at days 6 or 13 for cells containing either tubular (A, light gray bars), partially fragmented (B, dark gray bars) or fully fragmented (C, black bars) mitochondria. Oxygen consumption rate (OCR) is shown as absolute values in picomoles per minute in $G$ and as percentage normalized to basic respiration in $\mathrm{H}$ for cultures at 13 days after transduction with AAV-EGFP (dotted bars), AAV- $\alpha$-synuclein + EGFP (gray bars), AAV- $\beta$-synuclein + EGFP (black bars), and AAV- $\gamma$-synuclein + EGFP (hatched bars). OCR was recorded after consecutive application of oligomycin (Oligo), fluoro-carbonyl cyanide phenylhydrazone (FCCP) (Oligo + FCCP), and rotenone (Oligo + FCCP + rotenone), or after application of FCCP alone (FCCP w/o Oligo). (I) Motility of mitochondria at 13 days after transduction is given in pixels per second of live recording in neurites.

mitochondrial functionality in terms of motility, membrane potential, and energy production was not negatively influenced by $\beta S$.

\section{$\beta$ S Forms Ion-Conductive Transient Channels in Membranes}

Channel function of monomeric and oligomeric $\beta S$ was studied in conductance measurements with artificial membranes made of diphytanoyl phosphatidylcholine $/ n$-decane. Figure $5 \mathrm{~A}$ shows a typical current recording obtained when $\beta S$ monomers in a final concentration of $400 \mathrm{nM}$ were added to the cis-side of the membrane. Application of a voltage of $+100 \mathrm{mV}$ to the cis-side resulted in the formation of transient channels with a conductance of about 200pS (see Fig $5 \mathrm{~B})$ and a mean lifetime of about 1.8 seconds. $\beta \mathrm{S}$ oligomers also showed membrane activity, but this channel-forming activity was much less defined (see Supplementary Fig 6C), showing in particular intensive spontaneous spiking at many different levels. $\beta S$ fibrils could not be tested, because they contain residual detergents.

\section{Discussion}

Up to now, wild-type $\beta S$ had not been implicated in the etiology and progression of aggregopathies like PD. 

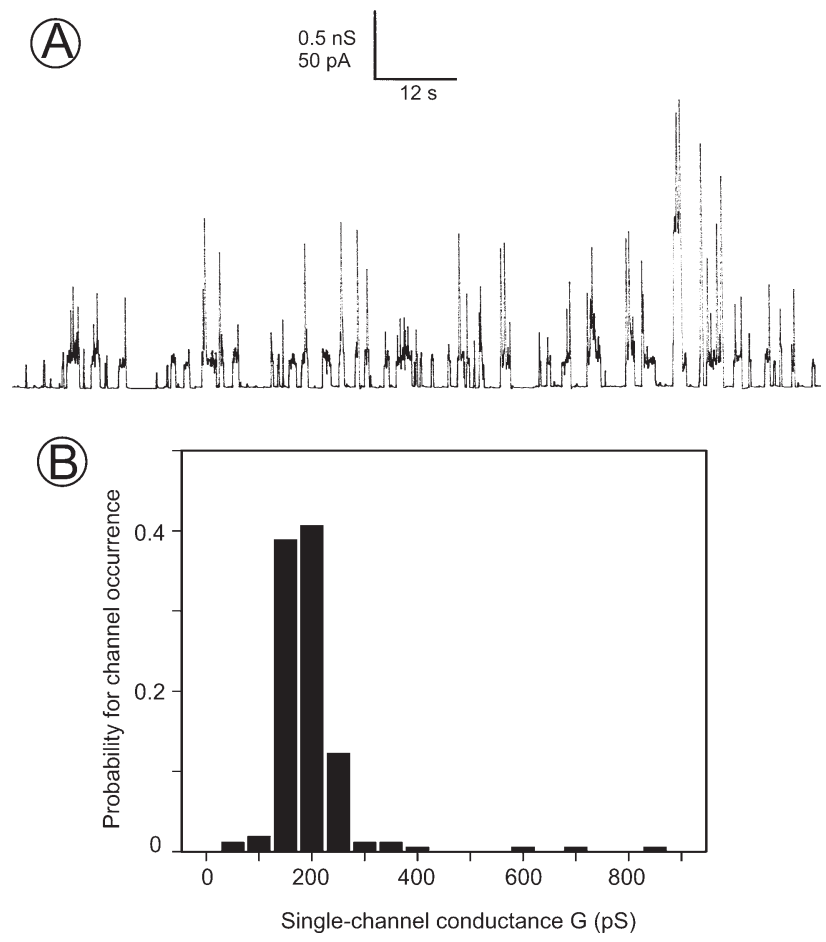

FIGURE 5: $\beta$-Synuclein forms transient channels in membranes. (A) Single-channel recording of a diphytanoyl phosphatidylcholine $(\mathrm{PC}) / \mathrm{n}$-decane membrane in the presence of $400 \mathrm{nM} \beta$-synuclein monomers added to the cis-side of the membrane. The aqueous phase contained $1 \mathrm{M} \mathrm{KCl}, \mathrm{pH} 6$. The membrane potential was $100 \mathrm{mV}$ applied to the cis-side of the membrane; $T=20^{\circ} \mathrm{C}$. (B) Histogram of the probability $P(G)$ for the occurrence of a given conductivity unit observed with membranes formed of $\mathrm{PC} / \mathrm{n}$-decane in the presence of $\beta$-synuclein. $P(G)$ is the probability that a given conductance increment $G$ is observed in the single-channel experiments. It was calculated by dividing the number of fluctuations with a given conductance increment by the total number of conductance fluctuations. The aqueous phase contained $1 \mathrm{M} \mathrm{KCl}, \mathrm{pH} 6$ and 400 to $800 \mathrm{nM} \beta$-synuclein. The applied membrane potential was $100 \mathrm{mV}$ on the cis-side; $\mathrm{T}=20^{\circ} \mathrm{C}$. The average single-channel conductance was $0.18 \mathrm{nS}$ for 162 single-channel events.

Rather, an at least partially neuroprotective role was suggested for $\beta S$, based on several studies demonstrating that $\beta S$ can ameliorate $\alpha S$-induced neurotoxicity in cell culture, transgenic animals, and viral vector-based models. $^{7,8}$

However, a contribution of $\beta S$, and putatively also of $\gamma S$, to neurodegenerative processes is emerging. First, in brains of sporadic PD and DLB patients, but not in control brains, $\beta S$ and $\gamma S$ could be detected in pathological presynaptic and axonal sites in the hippocampus. ${ }^{12}$ Second, the DLB-linked P123H mutation of $\beta$ S generated neuropathologic axonal swellings, astrogliosis, and memory deficits in transgenic mice. Crossing these mice with $\alpha \mathrm{S}$ transgenic mice caused significant loss of dopaminergic fibers in the striatum, indicating that the $\mathrm{P} 123 \mathrm{H}$ mutation of $\beta \mathrm{S}$ can cooperatively enhance neurodegeneration together with $\alpha \mathrm{S}{ }^{13}$ Finally, forced expression of $\gamma S$ caused robust neurodegeneration, mainly in the spinal cord of transgenic animals under control of the prion promoter, suggesting that $\gamma S$ has neurodegenerative capabilities as well. ${ }^{25}$

Our current results show that wild-type $\beta S$ forms PK-resistant aggregates in vivo. Compelling evidence suggests that PK-resistant aggregates consist, at least to a large extent, of fibrillar synuclein. $\alpha S$ fibrils generated in vitro are PK resistant, ${ }^{26-29}$ as is $\alpha \mathrm{S}$ in the detergent-insoluble fraction of human synucleinopathy brains. ${ }^{28,29}$ In contrast, small-sized oligomers are not PK resistant, as recently shown in brain lysates of A53T transgenic mice. ${ }^{30}$ Moreover, rationally designed nonfibrillating mutants of $\alpha S^{31}$ did not fibrillate in the test tube and did not form PK-resistant aggregates in dopaminergic neurons in vivo, despite being expressed to the same extent as fibrillating wild-type and A30P $\alpha S .^{16}$ Thus, the detection of PK-resistant $\beta S$ strongly argues for a robust fibrillation propensity of this protein in vivo, despite the lack of the NAC domain. Our data therefore confirm earlier in vitro studies, which demonstrated $\beta S$ fibrillation under conditions of macromolecular crowding or the presence of metal ions or pesticides. ${ }^{10}$

The extent of neurodegeneration induced by $\beta S$ in cultured nondopaminergic neurons was significantly less as compared to $\alpha S$, and $\beta S$ was able to partially ameliorate the toxicity of $\alpha \mathrm{S}$. In vivo, we found that 2 weeks overexpression of $\beta S$ resulted in less pronounced dopaminergic cell loss as compared to $\alpha \mathrm{S}$. However, after 8 weeks of overexpression of $\beta S$, cell loss was almost the same as that induced by $\alpha \mathrm{S}$. This finding indicates that either the dopaminergic phenotype or the accumulation of $\beta S$ over time renders the protein as neurotoxic as $\alpha S$ in a neuronal population of major importance for PD. Thus, the classification of $\beta S$ as a putatively neuroprotective counterpart to $\alpha S$ should be revisited. The relative expression levels of both proteins have to be considered in addition to their absolute expression levels, which may indeed vary between different studies conducted so far. Further investigations will also be necessary to determine why $\beta S$ causes less axonal degeneration than $\alpha \mathrm{S}$ in dopaminergic striatal projections.

A major neurotoxic mechanism attributed to $\alpha S$ and to idiopathic PD in general is mitochondrial pathology. ${ }^{32}$ Defects in mitochondrial respiratory chain components are reported in $\mathrm{PD}$ brains, ${ }^{33}$ mitochondrial pathology may precede neurodegenerative phenotypes in both Alzheimer disease and $\mathrm{PD},{ }^{34}$ and $\alpha \mathrm{S}$ induces mitochondrial fission and destruction independent of the normal DJ-1-dependent fusion/fission machinery. ${ }^{23}$ However, a recent study failed to prove that in nigral 
neurons of patients suffering from synucleinopathies, depletion of respiratory chain complex components in individual mitochondria takes place, but rather demonstrated a putative compensatory upregulation of these proteins. ${ }^{35}$ Under our experimental conditions, both $\alpha S$ and $\beta S$ induced structural mitochondrial lesions, but these destroyed mitochondria are functionally intact at least with regard to oxidative phosphorylation and ion homeostasis, until the affected neuron is about to die. These results suggest that structural deterioration of mitochondria, caused by both $\alpha \mathrm{S}$ and $\beta S$, is not necessarily causing an early and disease-initiating critical dysfunction, at least not in the time frame possible to investigate in cultured neurons. Given our demonstration of $\beta S$ being capable of forming ion-conductive membrane channels analogously to $\alpha \mathrm{S},{ }^{19,36}$ it was somewhat unexpected not to detect an impact of synucleins on mitochondrial ion homeostasis, suggesting that the mitochondrial membranes may not be affected by such channel-forming activity.

In conclusion, our results demonstrate that $\beta S$, so far classified as nonamyloidogenic, readily aggregated in dopaminergic neurons and caused an extent of neurodegeneration similar to $\alpha \mathrm{S}$. Thus, it seems necessary to take into account the degenerative properties of $\beta S$, which can no longer be considered only as a potentially protective scavenger of $\alpha S$-related neurotoxicity. Rather, the intrinsic toxic properties of $\beta S$ must be considered not only in DLB, where mutated $\beta S$ is linked to a familial form of the disease, but also in idiopathic PD, where alterations in $\beta S$ levels ${ }^{37}$ may be causatively involved in disease etiology and progression. Furthermore, the apparent link between aggregation propensity and neurotoxicity observed for $\alpha S$ and $\beta S$ versus $\gamma S$ suggests that, challenging the toxic oligomers hypothesis, fibrillation of synuclein could be a primary event triggering neurodegeneration.

\section{Acknowledgment}

This work was supported by the German Research Council-funded Center of Molecular Physiology of the Brain (G.T., S.K., M.B., T.F.O.) and by the German Research Council-funded project MI 685/2-1 (J.To., S.K.). M.Z. was supported by the Bundesministerium für Bildung und Forschung (NGFN-Plus 01GS08190). T.F.O. was supported by a Marie Curie International Reintegration Grant and an European Molecular Biology Organization installation grant. J.Te. was supported by the European Community\&s Seventh Framework
Programme FP7/2007-2013 under grant agreement $n^{\circ}$ HEALTH-2008-222925.

We thank U. Schöll and M. Zebski for excellent technical assistance, S. Mironov for help with calcium imaging, E. Gerhardt for the V5-tagged synucleins, K. Giller and $\mathrm{S}$. Wolf for preparation of $\beta$-synuclein samples, $\mathrm{E}$. Maier for black lipid membrane measurements, D. Riedel for electron micrographs, and L. Fonseca for circular dichroism data.

\section{Authorship}

G.T., J.To., and J.Te. contributed equally to this work.

\section{Potential Conflicts of Interest}

P.W., R.B., S.B., T.F.O., M.B., S.K.: grants/grants pending, German Research Council.

\section{References}

1. von Bohlen und Halbach $O$. Synucleins and their relationship to Parkinson's disease. Cell Tissue Res 2004;318:163-174.

2. Mori F, Tanji K, Yoshimoto M, et al. Immunohistochemical com parison of alpha- and beta-synuclein in adult rat central nervous system. Brain Res 2002;941:118-126.

3. Surgucheva I, McMahon B, Surguchov A. gamma-Synuclein has a dynamic intracellular localization. Cell Motil Cytoskeleton 2006;63: 447-458.

4. Greten-Harrison B, Polydoro M, Morimoto-Tomita M, et al. $\alpha \beta \gamma$ Synuclein triple knockout mice reveal age-dependent neurona dysfunction. Proc Natl Acad Sci U S A 2010;107:19573-19578.

5. Jellinger KA. Neuropathology of sporadic Parkinson's disease: evaluation and changes of concepts. Mov Disord 2012;27:8-30.

6. Uversky VN, Li J, Souillac P, et al. Biophysical properties of the synucleins and their propensities to fibrillate: inhibition of alphasynuclein assembly by beta- and gamma-synucleins. J Biol Chem 2002;277:11970-11978.

7. Hashimoto $M$, Rockenstein $E$, Mante $M$, et al. An antiaggregation gene therapy strategy for Lewy body disease utilizing beta-synuclein lentivirus in a transgenic model. Gene Ther 2004;11: 1713-1723.

8. Hashimoto $M$, Rockenstein $E$, Mante $M$, et al. beta-Synuclein inhibits alpha-synuclein aggregation: a possible role as an antiparkinsonian factor. Neuron 2001;32:213-223.

9. Fan Y, Limprasert P, Murray IV, et al. Beta-synuclein modulates alpha-synuclein neurotoxicity by reducing alpha-synuclein protein expression. Hum Mol Genet 2006;15:3002-3011.

10. Yamin G, Munishkina LA, Karymov MA, et al. Forcing nonamyloidogenic beta-synuclein to fibrillate. Biochemistry 2005;44: 9096-9107.

11. Vance JM, Ali S, Bradley WG, et al. Gene-environment interactions in Parkinson's disease and other forms of parkinsonism. Neurotoxicology 2010;31:598-602.

12. Galvin JE, Uryu K, Lee VM, Trojanowski JQ. Axon pathology in Parkinson's disease and Lewy body dementia hippocampus contains alpha-, beta-, and gamma-synuclein. Proc Natl Acad Sci U S A 1999:96:13450-13455.

13. Fujita M, Sugama S, Sekiyama K, et al. A beta-synuclein mutation linked to dementia produces neurodegeneration when expressed in mouse brain. Nat Commun 2010;1:110. 
14. Kügler S, Hahnewald R, Garrido M, Reiss J. Long-term rescue of a lethal inherited disease by adeno-associated virus-mediated gene transfer in a mouse model of molybdenum-cofactor deficiency. Am J Hum Genet 2007;80:291-297.

15. Shevtsova Z, Malik I, Garrido M, et al. Potentiation of in vivo neuroprotection by $\mathrm{BcIX}(\mathrm{L})$ and GDNF co-expression depends on post-lesion time in deafferentiated CNS neurons. Gene Ther 2006;13:1569-1578.

16. Taschenberger G, Garrido M, Tereshchenko Y, et al. Aggregation of $\alpha$ synuclein promotes progressive in vivo neurotoxicity in adult rat dopaminergic neurons. Acta Neuropathol 2012;123:671-683.

17. Kügler S, Meyn L, Holzmuller H, et al. Neuron-specific expression of therapeutic proteins: evaluation of different cellular promoters in recombinant adenoviral vectors. Mol Cell Neurosci 2001;17: 78-96.

18. Mironov SL, Skorova E, Hartelt N, et al. Remodelling of the respiratory network in a mouse model of Rett syndrome depends on brain-derived neurotrophic factor regulated slow calcium buffering. J Physiol 2009;587:2473-2485.

19. Kim HY, Cho MK, Kumar A, et al. Structural properties of poreforming oligomers of alpha-synuclein. J Am Chem Soc 2009;131: 17482-17489.

20. R Foundation for Statistical Computing. 2010. Available at: http:// www.R-project.org.

21. Rochet JC, Hay BA, Guo M. Molecular insights into Parkinson's disease. Prog Mol Biol Transl Sci 2012;107:125-188.

22. Correia SC, Santos RX, Perry G, et al. Mitochondrial importance in Alzheimer's, Huntington's and Parkinson's diseases. Adv Exp Med Biol 2012;724:205-221.

23. Nakamura K, Nemani VM, Azarbal F, et al. Direct membrane association drives mitochondrial fission by the Parkinson disease-associated protein alpha-synuclein. J Biol Chem 2011;286: 20710-20726.

24. Bueler H. Mitochondrial dynamics, cell death and the pathogenesis of Parkinson's disease. Apoptosis 2010;15:1336-1353.

25. Ninkina N, Peters O, Millership S, et al. Gamma-synucleinopathy: neurodegeneration associated with overexpression of the mouse protein. Hum Mol Genet 2009;18:1779-1794.
26. Conway KA, Harper JD, Lansbury PT Jr. Fibrils formed in vitro from alpha-synuclein and two mutant forms linked to Parkinson's disease are typical amyloid. Biochemistry 2000;39:2552-2563.

27. Giasson BI, Murray IV, Trojanowski JQ, Lee VM. A hydrophobic stretch of 12 amino acid residues in the middle of alpha-synuclein is essential for filament assembly. J Biol Chem 2001;276: 2380-2386.

28. Miake $H$, Mizusawa $H$, Iwatsubo $T$, Hasegawa M. Biochemical characterization of the core structure of alpha-synuclein filaments. J Biol Chem 2002;277:19213-19219.

29. Neumann M, Kahle PJ, Giasson BI, et al. Misfolded proteinase K resistant hyperphosphorylated alpha-synuclein in aged transgenic mice with locomotor deterioration and in human alpha-synucleinopathies. J Clin Invest 2002;110:1429-1439.

30. Tsika E, Moysidou M, Guo J, et al. Distinct region-specific alphasynuclein oligomers in A53T transgenic mice: implications for neurodegeneration. J Neurosci 2010;30:3409-3418.

31. Karpinar DP, Balija MB, Kügler S, et al. Pre-fibrillar alpha-synuclein variants with impaired beta-structure increase neurotoxicity in Parkinson's disease models. EMBO J 2009;28:3256-3268.

32. Schapira AH, Gegg M. Mitochondrial contribution to Parkinson's disease pathogenesis. Parkinsons Dis 2011;2011:159160.

33. Bueler $\mathrm{H}$. Impaired mitochondrial dynamics and function in the pathogenesis of Parkinson's disease. Exp Neurol 2009;218: 235-246.

34. Coskun P, Wyrembak J, Schriner SE, et al. A mitochondrial etiology of Alzheimer and Parkinson disease. Biochim Biophys Acta 2012;1820:553-564.

35. Reeve AK, Park TK, Jaros E, et al. Relationship between mitochondria and alpha-synuclein: a study of single substantia nigra neurons. Arch Neurol 2012;69:385-393.

36. Tosatto $\mathrm{L}$, Andrighetti AO, Plotegher $\mathrm{N}$, et al. Alpha-synuclein pore forming activity upon membrane association. Biochim Biophys Acta 2012;1818:2876-2883.

37. Beyer $\mathrm{K}$, Ispierto L, Latorre $\mathrm{P}$, et al. Alpha- and beta-synuclein expression in Parkinson disease with and without dementia J Neurol Sci 2011;310:112-117. 